September $\quad 6, \quad 2018 \quad 1: 59$ WSPC/INSTRUCTION $\quad$ FILE Sanchez'Javaloyes'Revised2

International Journal of Geometric Methods in Modern Physics

(C) World Scientific Publishing Company

\title{
Finsler metrics and relativistic spacetimes
}

\author{
Miguel A. Javaloyes \\ Departamento de Matemáticas, Universidad de Murcia, \\ Facultad de Matemáticas, Campus de Espinardo $s / n$ \\ Murcia, E-30100, Spain \\ majava@um.es \\ Miguel Sánchez \\ Departamento de Geometría y Topología, Universidad de Granada. \\ Facultad de Ciencias, Campus de Fuentenueva $s / n$. \\ E-18071 Granada (Spain) \\ sanchezm@ugr.es \\ Received (Day Month Year) \\ Revised (Day Month Year)
}

Submitted to the Special Issue for the XXII IFWGP Evora, with Associated Editors R. Albuquerque, M. de Leon and M. C. Munoz Lecanda.

Recent links between Finsler Geometry and the geometry of spacetimes are briefly revisited, and prospective ideas and results are explained. Special attention is paid to geometric problems with a direct motivation in Relativity and other parts of Physics.

Keywords: Finsler spacetime; Randers metrics; stationary spacetimes.

\section{Introduction}

There has been a recent interest in the links between Finsler Geometry and the geometry of relativistic spacetimes. A well-known reason comes from the viewpoint of applicability: Finsler metrics are much more general than Riemannian ones and, accordingly, if one replaces the Lorentz metric of a spacetime by a Finslerian counterpart, the possibility to model physical effects is richer. A more practical reason comes from a purely mathematical correspondence: the geometry of a concrete class of Finsler manifolds (Randers spaces) is closely related to the conformal structure of a class of spacetimes (standard stationary ones). So, results in one of these two fields can be translated into results on the other one - and, sometimes, this can be generalized to more general Finsler and Lorentz spaces. The purpose of this paper is to make a brief revision of this topic, emphasizing the applications to Physics.

After some preliminary definitions and motivations on Finsler metrics (Section 2), we focus on the problem of describing spacetimes by using Finsler elements 
(Section 3). We introduce and discuss a notion of Lorentz-Finsler metric which leads to (conic) Finsler spacetimes. Our definition is quite general and, so, several previous notions in the literature fit in it. Such a Finsler spacetime provides a cone structure. This notion has been studied systematically very recently [16], and it allows us to introduce classical Causality in the framework of Finsler spacetimes. In particular, some of the key results on this topic can be recovered, Theorem 1 It is worth pointing out: (a) the cone structure of a Finsler spacetime generalizes the one provided by the chronological futures of a classical spacetime, and defines implicitly a past cone structure (but no further assumption on reversibility should be necessary if one liked to include in the definition of conic Finsler spacetime the two cones at each point), and (b) in classical spacetimes, the cone structure is equivalent to the conformal one, but in Finsler spacetimes many non-conformally related Lorentz Finsler metrics will have the same cone structure and, thus, the same Causality - that is, the information of the Lorentz-Finsler metric not contained in the cone structure is much richer than in classical spacetimes.

In Section 4, we make a brief survey on the recent developments about the geometric correspondence between standard stationary spacetimes and Randers spaces. We focus in those questions with more clear applications to relativistic spacetimes and, so, topics such as the causal structure of standard stationary spacetimes or the gravitational lensing are emphasized. Finally, the conclusions are summarized in the last section.

\section{Finsler metrics and their applications}

Let us introduce the very general notion of (conic) pseudo-Finsler metric in a manifold $M$. Let $T M$ denote the tangent bundle of $M, \pi: T M \rightarrow M$, the natural projection and $A \subset T M \backslash \mathbf{0}$, an open subset satisfying that it is conic (that is, if $v \in A$ and $\lambda>0$, then $\lambda v \in A$ ), and it projects on all $M$ (i.e., $\pi(A)=M$ ). We say that a smooth function $L: A \subset T M \backslash \mathbf{0} \rightarrow \mathbb{R}$ is a (conic) pseudo-Finsler metric if $L$ is positive homogeneous of degree 2, namely, $L(\lambda v)=\lambda^{2} L(v)$ for any $v \in A$ and $\lambda>0$, and the fundamental tensor $g$, defined as the Hessian of $\frac{1}{2} L$ at every $v \in A$, is nondegenerate. In other words, given $v \in A$, the bilinear symmetric form in $T_{\pi(v)} M$ defined as

$$
g_{v}(u, w)=\left.\frac{1}{2} \frac{\partial^{2}}{\partial s \partial t} L(v+t u+s w)\right|_{t=s=0}
$$

for any $u, w \in T_{\pi(v)} M$, is non-degenerate.

Let us remark that classical Finsler metrics are a particular case of pseudoFinsler metrics. More specifically, $L$ is a Finsler metric if $A=T M \backslash \mathbf{0}$ and the fundamental tensor $g$ is positive definite for every $v \in A$. In this case, $L$ is always positive, since by homogeneity $L(v)=g_{v}(v, v)$ for every $v \in A$ and we can consider

${ }^{a}$ In this case, positive definiteness follows from non-degeneracy [25, Prop. 2.16 (ii)]. 
$F=\sqrt{L}$, which is positive homogeneous of degree one. The function $F$ is usually called the Finsler metric function, and it is determined by its indicatrix, i.e. the set of its unit vectors.

Among the most classical examples of Finsler metrics, we will use Randers metrics, which are given by

$$
F(v)=\alpha(v)+\beta(v)
$$

for every $v \in T M$, where $\alpha(v)=\sqrt{h(v, v)}$ and $h$ and $\beta$ are a Riemannian metric and a one-form in $M$ respectively. In fact, the fundamental tensor of $F$ is positive definite in $v \in T M$ if and only if $\alpha(v)+\beta(v)>0$ (see [25, Corollary 4.17]); this restriction is satisfied everywhere if the $h$-norm of $\beta$ is smaller than 1 , so defining a Randers metric. These metrics appear naturally in several contexts as in Zermelo navigation problem, which aims to describe the trajectories that minimize the time in the presence of a mild wind or current modelled by a vector field $W$. Then, these trajectories are given by geodesics of a Randers metric defined as

$$
F(v)=\frac{-g(v, W)+\sqrt{g(v, W)^{2}+g(v, v)(1-g(W, W))}}{1-g(W, W)}
$$

where $v \in T M, g$ is a Riemannian metric and $g(W, W)<1$ in all $M$ [2]. Observe that Zermelo metrics are always positive for every $v \in T M \backslash \mathbf{0}$ and then its fundamental tensor is positive definite. As we will see later, Randers metrics also appear naturally associated to stationary spacetimes describing their causal properties (see Section (4). Other remarkable example is given by Matsumoto metric, which describes trajectories minimizing time in the presence of a slope - recall that going up is slower than going down. This metric is defined as

$$
F(v)=\frac{\alpha(v)^{2}}{\alpha(v)-\beta(v)}
$$

for every $v \in T M$, and its fundamental tensor is positive definite in $v \in T M \backslash \mathbf{0}$ if and only if $(\alpha(v)-\beta(v))(\alpha(v)-2 \beta(v))>0$ [25, Corollary 4.15]. Therefore, Matsumoto metric is properly conic if this inequality is not satisfied by some $v \neq 0$. Randers and Matsumoto metrics are particular examples of the class of $(\alpha, \beta)$-metrics, which are defined as $F(v)=\alpha \phi(\beta(v) / \alpha(v))$, being $\phi$ an arbitrary non-negative real function (see 25] and references therein).

\section{Finsler-Lorentz metrics and spacetimes}

In classical General Relativity, a spacetime is a (connected, Hausdorff) n-manifold $M$ endowed with a Lorentzian metric $g$ and a time orientation, i.e., a continuous choice of one of the two timelike cones at each point, which will be regarded as future-directed. There are some speculative applications of the replacement of $g$ by a (generalization of a) Finsler metric $F$, as modeling possible anisotropies of the spacetime even at an infinitesimal level, or admitting speeds higher than light. In any case, the possibility to model a general action functional (homogeneous of 
degree two, but not necessarily coming from a quadratic form) justifies the study of the Lorentz-Finsler approach.

There are different possibilities in order to define a Finsler spacetime. Recall that, for a Riemannian metric $g_{R}$, the unit vectors constitute the indicatrix of a (standard) Finsler metric; in particular, each unit sphere $S_{p} \subset T_{p} M$ is convex (as the boundary of the unit ball) at each point. Nevertheless, if $g$ is a Lorentzian metric, one has to consider the subsets $S_{p}^{+}, S_{p}^{-} \subset T_{p} M$ containing, resp. the spacelike and timelike unit vectors at $p$. Notice that $S_{p}^{-}$always contains two connected parts, each one concave. For $n=2$ these properties also hold for $S_{p}^{+}$, but for higher dimensions $S_{p}^{+}$is connected and, at each point $p$, its second fundamental form (say, with respect to any auxiliary Euclidean product at $T_{p} M$ ) has Lorentzian signature. From the physical viewpoint, the most important elements of the spacetime are the causal vectors of the metric, since they model the trajectories of massive and massless particles. Observe in particular that the length of a causal curve $\gamma:[0,1] \rightarrow M$ in the spacetime is computed as

$$
\ell_{g}(\gamma)=\int_{0}^{1} \sqrt{-g(\dot{\gamma}, \dot{\gamma})} d s
$$

and a fundamental property in the spacetime is that causal geodesics locally maximize this length. If we want to define a more general way of measuring the length of curves (but preserving that the length does not depend on the parametrization of the curve and geodesics are local length-maximizers), then we must consider a positive one-homogeneous function $F: A \subset T M \backslash \mathbf{0} \rightarrow(0,+\infty)$ with fundamental tensor of signature $n-1$ (see [26]). This would be enough for timelike curves describing the trajectories of massive particles, but if in addition we want to describe the trajectories of massless particles, we will consider a positive two-homogeneous function $L$, rather than a one-homogeneous function, - since for a Lorentzian metric $g, \sqrt{-g(v, v)}$ is not smooth when $v$ is lightlike. Summing up, we say that a (conic) Finsler spacetime is a manifold endowed with a conic pseudo-Finsler metric $L: A \rightarrow[0,+\infty)$ which satisfies the following properties:

(i) each $A_{p}:=A \cap T_{p} M$ is convex in $T_{p} M$ (i.e., the segment in $T_{p} M$ connecting each two vectors $v_{p}, w_{p} \in A_{p}$ is entirely contained in $A_{p}$ ), in particular, each $A_{p}$ must be strictly included in a half-plane of $T_{p} M$,

(ii) $A$ has a smooth boundary in $T M \backslash \mathbf{0}$,

(iii) the extension of $L$ as 0 to the closure $\bar{A}$ of $A$ is smooth at $\hat{A}:=\bar{A} \backslash \mathbf{0}$ (notice that the extension is always continuous at 0 by homogeneity, and

\footnotetext{
b There are some non-trivial issues regarding smoothability. On the one hand, mathematically, it is well known that all Finsler metrics smoothly extendible to the 0-section must come from Riemannian metrics [45 Prop. 4.1]. On the other, physically, there are situations where it is natural to consider non-differentiable directions, so that one may allow this possibility explicitly 31. However, we will not go through these questions in the remainder (essentially, "smooth" might include some residual non-differentiable points with no big harm).
} 
this extension cannot be smooth there even for a classical Finsler metric, except if it comes from a Riemannian metric), and

(iv) both the fundamental tensor $g$ in (11) and its extension to the points in $\partial A \backslash \mathbf{0}$ have signature $n-1$.

For practical purposes, we will consider always that $L$ is extended to $\bar{A}$ and $g$ to $T_{v}\left(T_{p} M\right)$ for all $p \in M$ and $v \in \bar{A}_{p} \backslash \mathbf{0}$. In order to make computations related to lightlike geodesics, one can also assume that $L$ is smoothly extended (in a nonunique way) on a neighborhood of $\hat{A}$. We will call $F=\sqrt{L}$ the Lorentz-Finsler metric (defined on $\bar{A}$ ) of the Finsler spacetime. Observe that our notion of Finsler spacetime is based on a "conic" element which is essentially present in most of previous literature. In particular:

(1) In $[5$, the function $L$ is defined in all $T M$ and the fundamental tensor is assumed to have signature $n-1$. The restriction to one connected component of the subset $L^{-1}[0,+\infty) \backslash 0$ in every point $p \in M$ gives a (conic) Finsler spacetime as defined here. In [35131, the definition is essentially as in [5] with the opposite sign of $L$ (and an increasing attention to relax differentiability). The definition in [36] is somewhat more involved: they introduce an $r$-homogeneous function $L$, with $r \geq 2$ endowed with a fundamental tensor of signature one and, then, they associate a homogeneous Finsler function $F=\sqrt[r]{L}$. This allows one to deal with non-differentiability in cases that extend the lightlike vectors in our definition above. In [1], the author gives a definition analog to ours, but he excludes the conditions on the boundary (so that one is not worried about lightlike vectors).

(2) Lorentz-Finsler metrics appear in the context of Lorentz violation. Here, one starts with a background Lorentz-Minkowski space, but the movement of particles or waves is governed by a Lagrangian with a Lorentz-Finsler behavior (see [37]28]29] and references therein). Under natural hypotheses, the following positively homogeneous function is found in [27]:

$$
F(v)=m \sqrt{-g_{0}(v, v)}+g_{0}(v, a) \pm \sqrt{g_{0}(v, b)^{2}-g_{0}(b, b) g_{0}(v, v)},
$$

where $g_{0}$ is the standard metric of Minkowski spacetime $\mathbb{R}_{1}^{4}, v$ is any timelike vector and $a, b$ are two prescribed vector fields in $\mathbb{R}_{1}^{4}$. The interplay between $F$ and the background metric $g_{0}$ becomes important for physical applications (for example, the group velocity of propagating waves may exceed the light speed in $\mathbb{R}_{1}^{4}$ ). Even though $F$ can be regarded as a Lorentz-Finsler metric, in some cases the fundamental tensor of $F$ may be degenerate on some timelike directions (when $b=0$, it is easy to compute the fundamental tensor of $F$ 25. Proposition 4.17], and this becomes degenerate when $\left.F(v)=\sqrt{-g_{0}(v, v)}+g_{0}(v, a)=0\right)$.

(3) General physical theories of modified gravity (see 44] or the very recent articles 38139 and references therein) yield naturally Lorentz-Finsler spacetimes as defined above. The exact domain $A$ of $L$ for general expressions in Finsler-Cartan gravity (say, as in [39, formula (2)]) may be difficult to compute. However, 
one can compute $A$ easily in General Very Special Relativity, which possesses a simple Finslerian line element introduced by Bogoslovsky (say, the expression $F=\left(\sqrt{-g_{0}}\right)^{(1-b)} \omega^{b}$ in [30, formula (I.1)] becomes a Lorentz-Finsler metric with domain $A$ equal to the intersection at each point of the future time-cone of $g_{0}$ and the half-space $\omega>0$ ) or in Randers-type cosmologies as 4, formula (3.1)].

Notice that our definition of Finsler spacetime is a generalization of the structure obtained in a classical spacetime when one considers only its future causal cones. In the case of Lorentzian metrics, however, the value of the metric on the causal vectors is enough to determine the metric on all the vectors and, moreover, the lightlike vectors are enough to determine the metric up to a conformal factor. So, the cone structure for Lorentzian metrics is equivalent to the conformal structure. Nevertheless, this does not hold by any means in the case of (conic) Finsler spacetimes: clearly two Lorentz Finsler metrics $F, F^{\prime}$ on $M$ with the same domain $\bar{A}$ may not be equal up to a multiplicative function. However, the domain $A$ is a cone structure and, thus, one can reconstruct all the Causality Theory for Finsler spacetimes. Let us review this briefly.

Given a Finsler spacetime $(M, L)$ a tangent vector $v \in T M$ is called (futuredirected) timelike if $v \in A$, causal if $v \in \hat{A}$, lightlike if $v \in \hat{A} \backslash A$ and null if either $v$ is lightlike or the zero vectorl. A (piecewise smooth) curve $\gamma$ on $M$ is also called timelike, causal etc. depending on the character of its velocity at all the points. If $p, q \in M$, we say that $p$ lies in the chronological (resp. causal) past of $q$ if there exists a (future-directed) timelike (resp. causal or null) curve starting at $p$ and ending at $q$; in this case we write $p \ll q$ (resp. $p \leq q$ ) and we also say that $q$ lies in the chronological (resp. causal) future of $p$. The chronological and causal futures of $p$, as well as its corresponding pasts, are then defined formally as in the case of Lorentzian metrics:

$$
\begin{aligned}
& I^{+}(p)=\{q \in M: p \ll q\}, J^{+}(p)=\{q \in M: p \leq q\} ; \\
& I^{-}(p)=\{q \in M: q \ll q\}, J^{-}(p)=\{q \in M: q \leq p\} .
\end{aligned}
$$

One says also that a second Lorentz Finsler metric $F^{\prime}$ has cones wider than $F$, denoted $F \prec F^{\prime}$ when their corresponding domains $A, A^{\prime}$ satisfy $\hat{A} \subset A^{\prime}$. With these definitions, one can extend directly the causal ladder of classical spacetimes (see for example [33]) to Finsler spacetimes. We recall some of the steps of this ladder. A Finsler spacetime will be called chronological (resp. causal) when it does not admit closed timelike (resp. causal) curves, stably causal when there exists a causal Lorentz-Lorentz metric $F^{\prime}$ with wider cones $F \prec F^{\prime}$, and globally hyperbolic

\footnotetext{
"Notice that we have defined the Finsler metric only for "future-directed" causal directions, i.e., those in $\hat{A}$, and the past directions could be consistently regarded as those in $-\hat{A}$. It would be natural then to extend $L$ to $-\hat{A}$ by requiring full homogeneity $(L(v)=L(-v)$ for all $v \in-\hat{V})$, but this is not necessary for the issues regarding Causality. However such an extension (as well as possible further extension of $L$ to non-causal vectors) would be important for the particular physical theory one may be modelling.
} 
when it is stably causal and the following property holds: $J^{+}(p) \cap J^{-}(q)$ is compact for any $p, q \in M$. Trivially:

$$
\text { chronological } \Leftarrow \text { causal } \Leftarrow \text { stably causal } \Leftarrow \text { globally hyperbolic. }
$$

Taking into account the case of spacetimes, one realizes that there are more intermediate levels of the ladder as well as many subtle properties and relations among them to be considered for Finsler spacetimes. However, we focus here just on a pair of them, with deep implications for the global structure. To this aim, we define for a Finsler spacetime $(M, L)$ :

(a) a spacelike hypersurface is a smooth hypersurface $S$ such that no causal vector $v$ is tangent to $S$,

(b) a (spacelike) Cauchy hypersurface is a spacelike hypersurface $S$ such that any causal curve which is inextendible in a continuous way, intersects $S$ exactly once,

(c) a temporal function is a smooth function $t$ such that $d t(v)>0$ for all (futuredirected) causal vector $v$ (thus, its levels $t=$ constant are spacelike hypersurfaces) and,

(d) a Cauchy temporal function is a temporal function such that all its levels are Cauchy hypersurfaces.

In the case of classical spacetimes, results by Geroch [19] and Hawking [21] obtained at a topological level, plus their improvements to the smooth and metric cases by Bernal and Sánchez [7]8, prove the equivalence between being stably causal (resp, globally hyperbolic) and admitting a temporal (resp. Cauchy temporal) function. By using different arguments coming from KAM theory, these results were re-proved and extended to general cone structures by Fathi and Siconolfi [16]. So, as a consequence of the latter we get the following result.

Theorem 1. Let $(M, F)$ be a Finsler spacetime.

(1) If $(M, F)$ is stably causal, then it admits a temporal function (and, thus, it can be globally foliated by spacelike hypersurfaces).

(2) If $(M, F)$ is globally hyperbolic, then it admits a Cauchy hypersurface and a Cauchy temporal function.

Recall, however, that the Finsler spacetime has elements that are not characterized by the cone structure. For example, one can define a Finler separation $d(p, q) \in[0, \infty]$ between any $p, q \in M$ by taking the supremum of the lengths of the (future-directed) causal curves from $p$ to $q$-in a close analogous to the Lorentzian separation or distance for spacetimes with Lorentzian metric. The analogies and differences of $d$ with the Lorentzian case (in particular, its interplay with Causality) deserve to be studied further.

Remark 2. One expects that the converse of parts (1) and (2) of Theorem 1, as well as most of classical causality theory, hold. To achieve this, typical Lorentzian 
techniques, as those regarding limit curves or quasi-limits, must be adapted (recall the Lorentzian proofs of the converse in [19, [22, Sect. 6.6] or [34, Th. 14.38, Cor. 14.39]). Moreover, as the results in [16] work for very general cone structures, Theorem 1 can be applied to a more general class of spacetimes that includes those in [35. Example 3.3], which can be non-smooth in some timelike directions, and also the cone structure provided by the example (2) above (see [27]).

\section{Stationary to Randers correspondence and beyond}

From a classical viewpoint, a correspondence between some purely geometric elements of Lorentzian and Finslerian manifolds has been developed recently. Such a correspondence provides a precise description of certain objects in (classical) spacetimes in terms of an associated Finsler space. The precise correspondence is developed between a particular class of spacetimes, the standard completeconformastationary ones, or just stationary, for short, and a precise class of Finsler manifolds, the Randers ones (see (2)). But some consequences and techniques can be extrapolated to general Lorentzian and Finslerian manifolds.

We start with a trivial observation. Probably the simplest examples of Lorentzian manifolds are the products $\left(\mathbb{R} \times M, g_{L}=-d t^{2}+\pi^{\star} g_{0}\right)$, where $\pi$ : $\mathbb{R} \times M \rightarrow M$ and $t: \mathbb{R} \times M \rightarrow \mathbb{R}$ are the natural projections. The geometric properties of $\left(\mathbb{R} \times M, g_{L}\right)$ depend on those of $\left(M, g_{0}\right)$. In particular, each curve $(\mathbb{R} \supset) I \ni t \mapsto c(t) \in M$ parametrized with unit speed yields naturally two lightlike curves $t \rightarrow( \pm t, c(t))$ (future-directed with the sign "+" and past-directed with " - ", for the natural time orientation of the spacetime), which are geodesics iff $c$ is a geodesic in $\left(M, g_{0}\right)$. A less trivial spacetime is obtained if we admit cross terms between the time and space parts (independent of the time $t$ ). This can be described by using a 1-form $\omega$ on $M$, namely, we consider the spacetime:

$$
V=\left(\mathbb{R} \times M, g_{L}\right), \quad g_{L}=-d t^{2}+\pi^{\star} \omega \otimes d t+d t \otimes \pi^{\star} \omega+\pi^{\star} g_{0} .
$$

Now, we introduce the following Finslerian Fermat metrics associated to (4):

$$
F^{ \pm}=\sqrt{g_{0}+\omega^{2}} \pm \omega
$$

notice that these are metrics of Randers type, and $F^{-}$is the reverse Finsler metric of $F^{+}$, so, we will write simply $F$ for the latter and $\tilde{F}$ for the former. If we consider curves $c^{+}$and $c^{-}$in $M$ which are unit for $F$ and $\tilde{F}$ resp., the curves in the spacetime $t \rightarrow\left( \pm t, c^{ \pm}(t)\right)$ are again (future or past directed) lightlike curves in $V$, and each one is a geodesic up to parametrization iff so is the corresponding original curve $c^{+}$ or $c^{-}$(see the details in [12] or [13]). This suggests the possibility of describing the properties related to the Causality and conformal structure in (4) in terms of the geometry of the corresponding Randers space $(M, F)$.

It is worth pointing out that, as only conformally invariant properties will be taken into account, the class of spacetimes to be considered includes those conformal to (44). This class of spacetimes can be characterized intrinsically as those which 
are distinguishing (a causality condition less restrictive than strong causality) and admit a complete timelike conformal vector field. In fact, the conformal change of the metric $g$ by $-g / g(K, K)$, plus the result in [25], allows one to find the expression (4), which defines a normalized standard stationary spacetime. So, one will find a correspondence between the conformal properties of the elements in this class of metrics $\left(V, g_{L}\right)$ and the geometric properties of Randers spaces $(M, F)$. This has been carried out at different levels (see 910|11|12 13|18|20|24] or [23] for a review), and we will focus here in three of them, with clear physical applications.

\subsection{Causal structure}

Nicely, Fermat metrics allow one to determine the chronological and causal future and past of any point in the stationary spacetime (4). For example, if we take $(0, p) \in\{0\} \times M$ then the intersection of $I^{+}(0, p)$ with the slice $t=t_{0}$ of the spacetime is equal to $\left\{t_{0}\right\} \times B^{+}\left(p, t_{0}\right)$, where $B^{+}\left(p, t_{0}\right)$ is the open ball of center $p$ and radius $t_{0}$ for the Fermat metric $F\left(=F^{+}\right)$. From these considerations, one can describe in a precise way the causal structure of the spacetime. Concretely, one has 13:

Theorem 3. A stationary spacetime (4) is always causally continuous and it is

- Causally simple (i.e., it is causal, and the causal futures and pasts $J^{ \pm}(p)$ are closed) if and only if $(M, F)$ is convex, i.e. any pair $(p, q)$ in $M$ can be connected by means of an $F^{+}$-geodesic of length equal to the Finslerian distance $d_{F}(p, q)$.

- Globally hyperbolic if and only if the closed balls for the symmetrized distance of $d_{F}$ are compact.

- Globally hyperbolic with slices $t=$ constant that are Cauchy hypersurfaces if and only if $d_{F}$ is forward and backward complete.

Other causal elements which are described naturally with the Finslerian elements are the Cauchy horizons and developments. For example, given a subset $A \subset\{0\} \times M$, its future Cauchy horizon $H^{+}(A)$ is the graph, in $\mathbb{R} \times M$ of the function which maps each $y \in \bar{A}$ to $d_{F}(M \backslash A, y)$, i.e., the $d_{F}$-distance from the complement of $A$ to $y$.

Remark 4. The results in stationary spacetimes can also be translated to results in Randers metrics, sometimes generalizable to any Finsler manifold. Among them, we point out (see [13]):

- The theorem above suggests that the compactness of the closed symmetrized balls of $d_{F}$, which is a weaker hypothesis than the commonly used (forward or backward) completeness of $d_{F}$, can substitute the last hypothesis in many results. And, in fact, this is the case in classical theorems of Finsler Geometry, such as Myers' theorem or the sphere theorem.

- From the known fact that any globally hyperbolic spacetime admits a smooth spacelike Cauchy hypersurface, one can deduce that any Randers metric $R$ with 
compact symmetrized balls admits a trivial projective change $(R \rightarrow R+d f)$ such that the corresponding new Randers metric has the same pregeodesics as $R$, and it is forward and backward complete. This result has been extended by Matveev 32 for any Finsler metric.

- The results on horizons (a substantial topic in Lorentzian Geometry) in stationary spacetimes, yield directly results on Randers spaces. For example, the translation of a well-known result by Beem and Krolak [6 yields the following property for any subset $A$ of a Randers manifold $(M, R): p \in M$ is a differentiable point of the distance from $p$ if and only if it can be crossed by exactly one minimizing segment. This result has been extended to any Finslerian manifold by Sabau and Tanaka 42 .

\subsection{Visibility and gravitational lensing}

Assume that in our spacetime (4), a point $w$ represents an event and a line $l$, obtained as an integral curve of $\partial_{t}$, represents the trajectory of a stellar object. We wonder if there exist lightlike geodesics from $l$ to $w$ (i.e., whether an observer at $w$ can see the object $l$ ) and, in this case, if there are many of such geodesics (i.e., the existence of a lens effect such that $l$ is seen in two different directions). This situation is applicable to cosmological models such as Friedmann-Lemaitre-Robertson-Walker ones, as they are conformally stationary ( $l$ would represent a "comoving observer" of the model). The problem becomes more realistic if we choose some (open) region $\mathbb{R} \times D \subset \mathbb{R} \times M$ which contains $w$ and $l$, and search for geodesics contained in this region.

These problems are related to the convexity of $D$ for the Fermat metric (in the sense of the last subsection) which turns to be related to the convexity of its boundary $\partial D$. There are several different notions for the latter, as the local and infinitesimal convexity and, as shown in [3, they are equivalent to its geometric convexity. The latter means that, given any pair of points of $D$, any geodesic connecting them and contained in the closure $\bar{D}$, must be entirely contained in $D$. The (geometric) convexity of $D$ turns out equivalent to the light (geometric) convexity of the boundary of $\mathbb{R} \times D(\subset \mathbb{R} \times M)$ (i.e., the property of convexity holds when restricted to lightlike geodesics) and, finally, this is equivalent to the question of existence of connecting geodesics, yielding [10]:

Theorem 5. Assume that the closed balls of $\bar{D}$ computed for the restriction of the symmetrized distance $d_{F}^{s}$ are compact (which happens for example, if the intersections with $\bar{D}$ of the closed symmetrized balls in $M$ are compact or, simply, if $d_{F}$ is complete on all $M)$. Then, the following assertions are equivalent:

(i) $\left(D \times \mathbb{R}, g_{L}\right)$ is causally simple (i.e. $(D, F)$ is convex, Theorem $\left.\mathbf{3}\right)$.

(ii) $\partial D$ is convex for the Fermat metric $F$.

(iii) $\mathbb{R} \times \partial D$ is light-convex for the Lorentzian metric $g_{L}$.

(iv) Any point $w=\left(t_{p}, p\right) \in \mathbb{R} \times D$ and any line $l_{q}:=\{(\tau, q) \in \mathbb{R} \times D: \tau \in \mathbb{R}\}$, 
with $p \neq q$, can be joined in $\mathbb{R} \times D$ by means of a future-directed lightlike geodesic $z(s)=(t(s), x(s)), s \in[a, b]$, which minimizes the (future) arrival time $T=t(b)-t(a)$ (i.e., such that $x$ minimizes the $F$-distance in $D$ from $p$ to $q$ ).

(v) Idem to the previous property but replacing "future" by "past" and " $F$ distance" by (the reverse) " $\tilde{F}$-distance".

Remark 6. The previous result solves the question of existence of connecting lightlike geodesics. The question of multiplicity has some possibilities. First, the existence of a conjugate point of the lightlike geodesic $z$ at $w$. This is equivalent to the existence of a conjugate point at $p$ for its projection $x$ [11. Theorem 13], and it is regarded as trivial. Second, the non-triviality of the topology of $D$ may yield a topological lensing. In fact, one can prove that, whenever $D$ is not contractible, infinitely many connecting lightlike geodesics (with diverging arrival times) will exist.

The results can be also extended to the case of timelike geodesics, prescribing its length (i.e., the lifetime of the massive particles represented by such geodesics). The idea relies on a reduction of the problem to the lightlike one, by considering an extra spacelike dimension, see [13, Section 4.3] and [10, Section 5.2].

\subsection{Causal boundaries and further questions}

The studied stationary to Randers correspondence can be also applied to the study of the causal boundary of the spacetime in terms of Finsler elements. We recall that, in Mathematical Relativity, the Penrose conformal boundary is commonly used, in spite of the fact that it is not an intrinsic construction, and there are problems to ensure its existence or uniqueness. The causal boundary is a conformally invariant alternative, which is intrinsic and can be constructed systematically in any strongly causal spacetime (see [17] for a comprehensive study of this boundary). The computation of the causal boundary and completion of a stationary spacetime (4) has been carried out in full generality in [18. It must be emphasized that this boundary has motivated the definition of a new Busemann boundary in any Finslerian manifold. Even more, this has stimulated the study of further properties of the Gromov boundary in both, Riemannian and Finlerian manifolds.

It is also worth mentioning two further topics of current interest. The first one is the correspondence at the level of curvatures between the Weyl tensor of the stationary spacetime and the flag curvature of the Randers space, see [20. The second one is the possibility to extend the studied correspondence to the case of spacetimes with a nonvasnishing complete Killing vector field, whose causal type may change from timelike to lightlike and spacelike. Such metrics are related to a generalization of the Zermelo metrics which includes the possibility of a strong wind (i.e., with a speed higher than the one which can be reached by the engine of the ship), see [14]. 


\section{Conclusions}

As a first goal, we have introduced a very general notion of Finsler spacetime which includes many previous ones. This notion emphasizes the role of the cone structure, showing that many properties of (classical, relativistic) spacetimes are potentially transplantable to this setting -in particular, this holds for a major Lorentzian result such as Theorem 1. Remarkably, in relativistic spacetimes the cone structure is equivalent to the conformal structure and, thus, it is governed by the Weyl tensor. However, the metrics $F_{1}, F_{2}$ of two Finsler spacetimes with the same cone structure are not by any means necessarily conformal, and one is lead to deal directly with the cone structure. This cone structure would remain then at a more basic level than any possible Weyl-type tensor and, thus, also at a much basic level than the Cartan or Chern connections (of course, such connections will be relevant in order to describe concrete physical effects).

The importance on the choice of the connection is stressed because a Finsler metric admits several associated connections. Among them, one has the classical Cartan connection, the Berwald connection, the Chern-Rund connection and the Hashiguchi connection (see [40] or [41, §9.3]) . Thus, for example, two Finsler metrics with the same Chern connection may have different Cartan connections. As a consequence, the way to describe physical phenomena by using these connections may be non-equivalent and even controversial [15/43. Nevertheless, as emphasized here, all the possible physical causal influences will depend exclusively on the cone structure, at least at a non-quantum level.

As a second goal we have shown a different type of equivalence between the geometry of any Randers space and the conformal geometry of classical standard stationary spacetimes. This equivalence leads to obtain very precise results which relate: (a) the properties of distances on the Finslerian side with the Causality on the side of the spacetime, (b) the properties related to Finslerian convexity wth the properties related to relativistic observability and lensing, (c) accurate relations between ideal Finslerian boundary and the causal boundary (and, implicitly then, the conformal one) of spacetimes, or (d) interesting relations between Finslerian curvature and Weyl curvature [20. This equivalence has suggested new results in both, Finslerian and Lorentzian geometries. Moreover, a forthcoming work [14] will show its extendability and applicability to new fields - namely, an extension of classical Finsler metrics which allows one to model strong winds in Zermelo navigation problem is introduced, and its geometrical properties are shown equivalent to the conformal ones of a class of spacetimes wider than standard stationary ones, which allow the possibility of horizons and black holes.

\section{Acknowledgments}

Comments by Kostelecki, Laemmerzahl, Perlick, Szilasi and the referee are warmly acknowledged. Both authors are partially supported by the Grant P09-FQM4496 (J. Andalucía) with FEDER funds. The first-named author is also partially 
supported by MINECO-FEDER project MTM2012-34037 and Fundación Séneca project $04540 /$ GERM/06, Spain. This research is a result of the activity developed within the framework of the Programme in Support of Excellence Groups of the Región de Murcia, Spain, by Fundación Séneca, Regional Agency for Science and Technology (Regional Plan for Science and Technology 2007-2010). The secondnamed author acknowledges the support of IHES, Bures sur-Yvette, France, for a three months stay in 2013 (which included the period of celebration of the meeting IFWGP); he is also partially supported by MTM2010-18099 (MICINN-FEDER).

\section{References}

[1] G. S. Asanov, Finsler geometry, relativity and gauge theories (Fundamental Theories of Physics D. Reidel Publishing Co., Dordrecht, 1985).

[2] D. Bao, C. Robles and Z. Shen, Zermelo navigation on Riemannian manifolds, J. Differential Geom., 66 (3) (2004), 377-435.

[3] R. Bartolo, E. Caponio, A. V. Germinario and M. Sánchez, Convex domains of Finsler and Riemannian manifolds, Calc. Var. Partial Differential Equations, 40(3-4) (2011), 335-356.

[4] S. Basilakos and P. Stavrinos, Cosmological equivalence between the Finsler-Randers space-time and the DGP gravity model, Phys. Rev. D 87043506 (2013).

[5] J. K .Beem, Indefinite Finsler spaces and timelike spaces, Canad. J. Math., 22 (1970), 1035-1039.

[6] J. K .Beem and A . Królak, Cauchy horizon end points and differentiability, J. Math. Phys., 39(11) (1998), 6001-6010.

[7] A. N. Bernal and M. Sánchez, On Smooth Cauchy Hypersurfaces and Geroch's Splitting Theorem, Commun. Math. Phys., 243 (2003), 461-470.

[8] A. N. Bernal and M. Sánchez, Smoothness of time functions and the metric splitting of globally hyperbolic spacetimes, Commun. Math. Phys., 257 (2005), 43-50.

[9] L. Biliotti and M. Á. Javaloyes, $t$-periodic light rays in conformally stationary spacetimes via Finsler geometry, Houston J. Math. 37(1) (2011), 127-146.

[10] E. Caponio, A.V. Germinario and M. Sánchez, Convex regions of stationary spacetimes and Randers spaces. Applications to lensing and asymptotic flatness, arXiv:1112.3892 [math.DG].

[11] E. Caponio, M. Á. Javaloyes and A. Masiello, Morse theory of causal geodesics in a stationary spacetime via Morse theory of geodesics of a Finsler metric, Ann. Inst. H. Poincaré Anal. Non Linéaire, 27(3) (2010), 857-876.

[12] E. Caponio, M. Á. Javaloyes and A. Masiello, On the energy functional on Finsler manifolds and applications to stationary spacetimes, Math. Ann., 351(2) (2011), 365392.

[13] E. Caponio, M. Á. Javaloyes and M. Sánchez, On the interplay between Lorentzian Causality and Finsler metrics of Randers type. Rev. Mat. Iberoamericana, 27(3) (2011), 919-952.

[14] E. Caponio, M. Á. Javaloyes and M. Sánchez, Wind Finslerian structures: from Zermelo's navigation to the causality of spacetimes, arXiv:1407.5494 [math.DG].

[15] Z. Chang and X. Li, Modified Friedmann model in Randers-Finsler space of approximate Berwald type as a possible alternative to dark energy hypothesis Phys. Lett. B, 676 (2009) 173-176.

[16] A. Fathi and A. Siconolfi, On smooth time functions, Math. Proc. Cambridge Philos. Soc., 152(2) (2012), 303-339. 
[17] J. L. Flores, J. Herrera and M. Sánchez, On the final definition of the causal boundary and its relation with the conformal boundary, Adv. Theor. Math. Phys., 15(4) (2011), 991-1057.

[18] J. L. Flores, J. Herrera and M. Sánchez, Gromov, Cauchy and causal boundaries for Riemannian, Finslerian and Lorentzian manifolds, Mem. Amer. Math. Soc. 226 (2013), No. 1064 (77 pp).

[19] R. Geroch, Domain of dependence, J. Math. Phys. 11 (1970), 437-449.

[20] G. W. Gibbons, C. A. R. Herdeiro, C. M. Warnick and M. C. Werner, Stationary metrics and optical Zermelo-Randers-Finsler geometry, Phys. Rev. D, 79(4) (2009), $044022,21$.

[21] S. W. Hawking, The existence of cosmic time functions. Proc. Roy. Soc. London, series A 308 (1969), 433-435.

[22] S.W. Hawking and G.F.R. Ellis, The large scale structure of space-time, Cambridge University Press, Cambridge, 1973.

[23] M. Á. Javaloyes, Conformally Standard Stationary Space-Times and Fermat Metrics, Recent Trends in Lorentzian Geometry, Springer Proceedings in Mathematics 8 Statistics 26 (2013), 207-230.

[24] M. Á. Javaloyes, L. Lichtenfelz and P Piccione, Almost isometries of non-reversible metrics with applications to stationary spacetimes, arXiv:1205.4539v2 [math.DG].

[25] M. Á. Javaloyes and M. Sánchez, On the definition and examples of Finsler metrics, Ann. Sc. Norm. Super. Pisa Cl. Sci, (5) Vol XIII (2014), 1-46.

[26] M. Á. Javaloyes and H. Vitório, Zermelo navigation in pseudo-Finsler metrics, in progress.

[27] V. A. Kostelecky, Riemann-Finsler geometry and Lorentz-violating kinematics, Phys. Lett. B, 701(1) (2011), 137-143.

[28] V. A. Kostelecky and N. Russell, Classical kinematics for Lorentz violation, Physics Letters B, 693 (2010) 443-447.

[29] V. A. Kostelecky, N. Russell and R. Tso, Bipartite Riemann-Finsler Geometry and Lorentz Violation, Physics Letters B, 716 (2012) 470-474.

[30] A.P. Kouretsis, M. Stathakopoulos and P.C. Stavrinos: General very special relativiy in Finsler cosmology, Phys. Rev D, 79:104011 (2009).

[31] C. Laemmerzahl, V. Perlick and W. Hasse, Observable effects in a class of spherically symmetric static Finsler spacetimes, Phys Rev D, 86:104042 (2012).

[32] V. Matveev, Can We Make a Finsler Metric Complete by a Trivial Projective Change?, Recent Trends in Lorentzian Geometry, Springer Proceedings in Mathematics 85 Statistics 26 (2013), 231-242.

[33] E. Minguzzi and M. Sánchez, The causal hierarchy of spacetimes, in Recent developments in pseudo-Riemannian geometry, ESI Lect. Math. Phys., Eur. Math. Soc., Zürich (2008) 299-358.

[34] B. O'Neill, Semi-Riemannian geometry. With applications to relativity, in 'Pure and Applied Mathematics', 103, Academic Press, N.Y. (1983).

[35] V. Perlick, Fermat principle in Finsler spacetimes, Gen. Relativity Gravitation, 38(2) (2006), 365-380.

[36] C. Pfeifer and M. Wohlfarth, Causal structure and electrodynamics on Finsler spacetimes, Phys. Rev. D 84:044039 (2011).

[37] N. Russell, Remarks on Finsler Geometry and Lorentz Violation, Proceedings of the Sixth Meeting on CPT and Lorentz Symmetry, V.A. Kostelecky (Editor), World Scientific, Singapore, 2014.

[38] P.C. Stavrinos and S. Vacaru, Cyclic and Ekpyrotic Universes in Modified Finsler Osculating Gravity on Tangent Lorentz Bundles, Class. Quant. Grav. 30055012 (2013). 
September

6 , Sanchez'Javaloyes'Revised2

[39] P. Stavrinos, O. Vacaru and S. Vacaru, Off-Diagonal Solutions in Modified Einstein and Finsler Theories on Tangent Lorentz Bundles, arXiv:1401.2879 [physics.gen-ph].

[40] J. Szilasi and R. Lovas, Some aspects of differential theories. Handbook of global analysis, 1069-1114, 1217, Elsevier Sci. B. V., Amsterdam, 2008.

[41] J. Szilasi, R. L. Lovas and D. Cs. Kertesz, Connections, Sprays and Finsler Structures, World Scientific, 2014.

[42] M .Tanaka and S.V. Sabau, The cut locus and distance function from a closed subset of a Finsler manifold, arXiv:1207.0918v3 [math.DG].

[43] S. Vacaru, Critical Remarks on Finsler Modifications of Gravity and Cosmology by Zhe Chang and Xin Li Phys. Lett. B 690 (2010) 224-228.

[44] S. Vacaru, Principles of Einstein-Finsler Gravity and perspectives in Modern Cosmology Int. J. Modern Phys. D 21 No. 9 (2012) 1250072.

[45] F.W. Warner, The conjugate locus of a Riemannian manifold, Amer. J. Math. 87, (1965) 575-604. 